\title{
Rhythmic Auditory Stimulation (RAS) and Motor Rehabilitation in Parkinson's Disease: New Frontiers in Assessment and Intervention Protocols
}

\author{
Mauro Murgia ${ }^{1,2}$, Federica Corona ${ }^{3}$, Roberta Pili ${ }^{4}$, Fabrizio Sors $^{2}$, Tiziano Agostini ${ }^{2}$, Carlo Casula $^{5}$, \\ Massimiliano Pau ${ }^{3}$ and Marco Guicciardi ${ }^{1, *}$
}

\author{
${ }^{I}$ Department of Pedagogy, Psychology, Philosophy, University of Cagliari, Italy; ${ }^{2}$ Department of Life Sciences, Univer- \\ sity of Trieste, Italy; ${ }^{3}$ Department of Mechanical, Chemical and Materials Engineering, University of Cagliari, Italy; \\ ${ }^{4}$ Department of Surgical Sciences, University of Cagliari, Italy; ${ }^{5}$ Physical Medicine and Rehabilitation Service, \\ "G.Brotzu" General Hospital, Cagliari, Italy
}

\begin{abstract}
Previous studies have demonstrated that physical therapy accompanied by Rhythmic Auditory Stimulation (RAS) can improve the motor skills of patients with Parkinson's disease and, in particular, their gait disturbances. In the present work we describe the neurological bases and perceptual-motor deficits generally associated with Parkinson's disease, with a specific focus on gait disturbances. Within this framework, we review the role of auditory cueing in the modulation of patients' gait, addressing this issue from the cognitive, neurological and biomechanical perspectives. In particular, we focus on the new frontiers of both assessment and intervention. With regards to the assessment, we describe the advantages of the three-dimensional quantitative multifactorial gait analysis. As concerns the intervention, we illustrate the potential impact of the administration of ecological footstep sounds as rhythmic cues.
\end{abstract}

Keywords: Gait, Parkinson's disease, rehabilitation, rhythmic cues, sound.

\section{INTRODUCTION}

Parkinson's disease (PD) is a chronic progressive neurodegenerative disorder whose symptoms consist of the gradual loss of motor and non-motor functions [1]. The motor symptoms are the most relevant ones and consist of bradykinesia, rigidity, tremor, postural instability, and gait disturbance, usually associated with an increased probability of falls. Among the non-motor symptoms, previous studies report hyposmia (altered sense of smell), depression, cognitive decline, psychiatric and sleep disorders [2]. It appears that some non-motor symptoms may occur early in the course of the disease, even at a premotor stage [3, 4], contrary to previous beliefs that PD was solely a disorder of movement [1]. However, from the patient's point of view, the loss of motor functions probably remains the most challenging issue to deal with in daily activities, since it affects overall quality of life.

From a pathophysiological perspective, a characteristic of $\mathrm{PD}$ is the progressive degeneration of dopaminergic neurons in basal ganglia and, in particular, in the substantia nigra pars compacta, although the pathological processes in PD do not regard only the dopaminergic system [5]. Indeed, empirical evidence suggests that PD is a more diffuse pathology involving other non-dopaminergic systems, such as the serotonergic, noradrenergic, glutamatergic, and cholinergic systems, within cortical, brainstem, and basal ganglia regions

*Address correspondence to this author at the Department of Pedagogy, Psychology, Philosophy, University of Cagliari, Via Is Mirrionis 1, 09123 Cagliari, Italy; Tel: +39 0706757518; Fax: +39 0706757291;

E-mail: marco.guicciardi@unica.it
[1, 6-9]. To cope with altered levels of neurotransmitters, pharmacological treatments such as levodopa, non-ergot dopamine agonists, and MAO-B inhibitors are commonly used. Moreover, other non-pharmacological approaches such as physical therapy, accompanied by regular exercise and an active lifestyle, are also effective in the treatment of motor symptoms $[2,10]$.

Among the aforementioned motor symptoms, gait disturbance probably represents the main impairment of PD patients. In fact, they are characterized by a typical shortstepped, narrow based, shuffling gait, and experience difficulties in adjusting gait parameters to meet changing task demands [11]. Despite the success of pharmacological treatments in improving some features of PD, in some cases gait deficits can be resistant to medication and over time become one of the most debilitating symptoms $[12,13]$. Therefore, the attention of many scientists has focused on investigating alternative therapies as well. Among the nonpharmacological approaches, one of the methods most studied is Rhythmic Auditory Stimulation (RAS). This method consists of the administration of auditory cues that provide patients with a rhythmic cadence, thus facilitating their gait regulation. The effectiveness of RAS has often been assessed with quantitative movement analysis techniques, in some cases by exploiting state-of-the-art technologies of motion capture systems which in a single session integrate data associated with kinematics, kinetics and muscular activation during gait, and which are able to detect even subtle improvements in gait patterns.

The present work reviews the mechanisms underpinning the RAS method and the main results obtained in previous 
studies. Moreover, we illustrate the most recent and advanced approaches both for the quantitative assessment of gait in PD patients and for their gait modulation through the RAS method.

\section{EMPIRICAL EVIDENCE SUPPORTING RHYTH- MIC AUDITORY STIMULATION IN PD}

\subsection{Timing Mechanisms in PD}

Skill in walking is based on the precise coordination of muscle activation. In other words, it is founded on the correct functioning of timing mechanisms which - like an orchestra conductor - coordinate and direct every movement of our body. The regulation of these timing mechanisms in humans is performed by different brain areas. Among these, much empirical evidence indicates that basal ganglia play a very important role [14]. The involvement of basal ganglia in timing processing has been demonstrated in healthy participants through functional imaging studies [15-19], and in clinical studies in PD patients [20-23], whose basal ganglia are particularly affected.

From a cognitive perspective, when PD patients are in their "off" state, that is, not under the effect of medication, they experience deleterious effects on their temporal processing capabilities compared to healthy subjects. In particular, they show dysfunction in repetitive motor timing [20], they have a slower "internal clock" [21], and their response pattern violates the normal scalar property [24]. The dysfunction of temporal processing appears to be one origin of gait disturbance $[13,14,25]$. In other words, a deficient timing process may result in an irregular timing of walking pace and, as a consequence, impaired motor performance [13].

\subsection{Effects of Rhythmic Auditory Stimulation on Motor Performance}

In the early 1990s, Thaut and colleagues successfully tested a new motor rehabilitation method based on auditory cueing in stroke patients with hemiparetic gait [26]. The success of the auditory cueing method with stroke patients stimulated investigations regarding its extension to PD patients [27]. In the subsequent years, Thaut and collaborators used their cueing method - named Rhythmic Auditory Stimulation - to improve the gait deficits of $\mathrm{PD}$, providing patients with rhythmic guidance to facilitate patients' walking pace by influencing their internal timing mechanisms $[28,29]$.

In one of their first studies on PD [29], Thaut and colleagues compared the data of PD patients who were randomly assigned to one of the following three conditions: 1) RAS training; 2) internally self-paced training; 3) no training. The training programs lasted three weeks and consisted of 30 minutes of daily exercise: Walking on a flat surface, stair stepping and stop and go exercises. The participants assigned to the RAS condition performed the exercises with auditory stimulation at three different tempos (normal, quick and fast) every day. The tempos were progressively increased by 5 to $10 \%$ in the second week, and by an additional 5 to $10 \%$ in the third week. Participants assigned to the internally self-paced training condition performed exactly the same exercises, without RAS (they were instructed to exercise at different speeds similar to those of the RAS training patients). The results showed that both RAS and selfpaced training patients improved their gait parameters. However, the improvements of the RAS training patients in gait velocity, stride length, and step cadence were significantly greater than both self-paced training and no-training patients. Moreover, the RAS training participants had a significant reduction in amplitude variability in the anterior tibialis and vastus lateralis muscles. A more in-depth analysis of electromyography patterns confirmed these results, evidencing significant decreases in tibialis anterior shape variability and asymmetry, and gastrocnemius shape variability after threeweeks of RAS training [28].

The studies described above aroused much enthusiasm among researchers and stimulated further investigations on the RAS method in PD, with the aim of extending results and better exploring the mechanisms underpinning the method. Some of these studies investigated the immediate effects of RAS on motor performance by assessing motor parameters after a single session of RAS [30-48]. Other studies explored the mid-/long-term effects of RAS training, varying different parameters, such as number of training weeks, number of sessions per week, session duration, kind of stimuli, kind of exercise, administered tempos, severity of participants' disease [42, 49-59].

The majority of these studies reported positive effects of RAS both immediately after a single RAS session and after a longer training program. Indeed, in both cases, it is quite well-established that RAS improves gait velocity, cadence, and stride length $[43,50-52,57,60]$. Moreover, most of the studies reported improvements regarding the symmetry of muscle activation in legs and arms, as well as timing variability $[28,33,35,61]$ and, in general, stability while walking $[13,62]$.

From a clinical perspective, it has been reported that RAS training can facilitate performance improvements relative to the Dynamic Gait Index and Tinetti Test, and that these improvements persisted at least four weeks after practice termination [54]. Moreover, some studies reported a decreased worry about falling, assessed through the Falls Efficacy Scale [57], and improvements in the activities of daily life and motor subscales of the Unified Parkinson's Disease Rating Scale [54, 56, 63], especially in the most severe patients [30].

Another important aspect regards the effects of RAS on "freezing", a transient episode in which the motor activity being attempted by an individual is halted [64]. Some studies have reported positive effects of RAS on freezing by assessing it through objective measures $[31,34,57]$ and subjective measures, such as the Freezing of Gait Questionnaire [54]. However, other studies failed to find improvements on freezing [49] or found that after RAS patients with freezing may even experience stride length decreases $[13,46]$. In our opinion, further research is needed to better understand the effects of RAS on freezing.

\subsection{Auditory Cues Versus Other Modalities}

The effects of rhythmic cues on motor production have been studied in the domain of neurosciences and cognitive 
psychology. From a neurophysiological perspective, many studies have demonstrated that listening to auditory rhythms determines the activation of motor regions of the brain, such as the supplementary motor area, midpremotor cortex, cerebellum and basal ganglia [65-67]. Such evidence suggests the existence of a functional connection between motor and acoustic areas of the brain concerning the elaboration of rhythmic material. For this reason, from a cognitive perspective, the processing of rhythmic material is more accurate in the auditory modality compared to other sensorial modalities $[68,69]$. For instance, many studies by Repp and colleagues demonstrated that sensorimotor synchronization in tapping tasks is more accurate with auditory rhythms than with visual rhythms [70], and that auditory distractors attract participants' taps more than visual distractors [71].

The superiority of auditory stimulation compared to other modalities, such as visual and tactile, has been supported by a systematic review on rhythmic stimuli in PD [63]. Lim and colleagues analyzed twenty-four studies and concluded that insufficient evidence was found for improving gait of PD patients with the help of visual cueing, tactile cueing or a combination of auditory and visual cueing. On the contrary, they claimed that the walk of PD patients can be positively influenced by auditory rhythmic cueing.

\subsection{How Does Rhythmic Auditory Stimulation Work?}

One of the main disturbances of PD patients is the difficulty in performing automatized movements $[13,59]$. These movements are usually coordinated by the basal ganglia which, in normal conditions, guarantee the execution of submovements within an automatized sequence [72]. In PD patients the functioning of the internal clock that coordinates the automatic execution of these sub-movements is somehow compromised. For this reason, automatic cycling movements, such as walking, become over time more difficult with the progression of the disease.

The function of RAS is to substitute or at least assist the deficient internal clock of PD patients. RAS provides external timing that acts as an internal clock, thus facilitating the regulation of patients' internal timing $[23,73]$. As a consequence, the administration of auditory rhythms helps patients during the execution of automatized movements (pacing their walk, for instance) and determining better motor outcomes. In cognitive terms, this process is described by Thaut and Abiru as an oscillator-entrainment system, where the rhythmic processes in neural motor networks become entrained to rhythmic timekeeper networks in the auditory system [27]. In this model the timekeeper networks are thought to be driven peripherally from auditory rhythmic inputs.

Neurophysiological studies suggests that within the basal ganglia the putamen is particularly affected in PD [8], while it is responsive to rhythmic stimuli [66]. Functional imaging studies have shown severe reductions in putamen and related cortical and cortico-striatal activity during self-initiated or self-paced movements, while this activity is less compromised during externally paced ones [74-76]. Thus, the putamen may be one of the brain areas that are positively affected by RAS.

\section{NEW APPROACHES FOR ASSESSMENT: THREE- DIMENSIONAL QUANTITATIVE GAIT ANALYSIS}

The term "Gait Analysis" (GA) refers to the application of quantitative three-dimensional multifactorial analyses of human movement to the specific case of locomotion. In particular, GA encompasses kinematics (i.e. joint angular displacements), kinetics (ground reaction forces, joint moments and powers) and muscular activation (by means of surface Electromyography, sEMG) aspects of the locomotor pattern, supplying at the same time information about its main temporal (velocity, cadence, and gait cycle duration) and spatial (step length and width) parameters. The subdivision between stance, swing and double support phases is also available once the gait cycle has been identified using manual or automatic approaches.

\subsection{Gait Kinematics in Individuals with PD}

Although many studies have attempted to quantitatively characterize gait patterns in individuals affected by PD, it is to be noted that most of them focus on the study of spatiotemporal parameters, while few consider and analyze kinematics, kinetics and muscular activation data.

The early pioneering studies on gait kinematics in PD were performed in the 1970s using simple photographic techniques [77-79]. After placing a number or reflective targets in the shape of strips on specific anatomic landmarks, the patient was photographed (at time intervals in the range $0.05-0.10 \mathrm{~s}$ ) and the photos processed to extract the angular displacement patterns in hip, knee and ankle joint, the patterns of flexion-extension of the upper limbs, the transverse rotation of the pelvis and thorax and the vertical trajectories of the head, as well as the main spatio-temporal parameters of the gait. Although intrinsically limited by the bidimensional nature of the acquired data, these studies were the first to recognize the importance of objective gait analysis in characterizing the specific features of gait in PD. Such features, even though easily recognizable by an expert clinician, might be subject to changes (either related to the progression of the disease or to specific pharmacologic and rehabilitative treatments) that are difficult to assess without the support of quantitative parameters.

Nevertheless, further significant advancements in GA studies in PD occurred only in the mid-1990s, when more sophisticated technologies providing three-dimensional information on human motion (e.g. optoelectronic stereophotogrammetry) became accessible at reasonable costs. In this technique, spherical passive reflective markers are placed on the lower extremities, pelvis, and trunk of the subject according to standardized protocols [80] and, during walking, the three-dimensional trajectories of the markers are captured by a certain number of high-frequency cameras (typically six or more with frequencies ranging from 50 to $240 \mathrm{~Hz}$ ) which also provide stroboscopic infrared illumination. Such data are then processed by a workstation to provide the kinematic parameters related to the body districts of interest, namely trunk, pelvis, thigh, shank, and foot. The final output of the GA is usually represented by a series of diagrams that show the variation of angular displacements (pelvic tilt, rotation and obliquity, hip flexion-extension, adduction-abduction 
and rotation, knee flexion-extension, ankle dorsiflexion, and foot progression) within the gait cycle.

To date, kinematic data coming from three-dimensional GA have proved to be very useful not only to basically characterize the distinctive features of gait patterns in PD, but also to objectively and quantitatively assess the effects of neurosurgery, electrical stimulation, pharmacological and rehabilitative treatments $[11,81-91]$.

\subsection{Summary of Measures of Gait Kinematics}

Generally speaking, the large amount of data originated by GA may result complex to interpret for the clinician, and therefore not fully suitable to allow an easy and rapid assessment of the patient's functional limitation. This fact may represent an obstacle when, in clinical practice, the most significant measures have to been identified in a reasonable time.

Hence, researchers have attempted to summarize the overall quality of gait pattern with single concise measures which, with a single value, condense the whole set of kinematic data obtained by GA and thus make data interpretation easier. A detailed overview of the most widespread gait summary measures is reported in the paper by Cimolin and Galli [92].

Among the several proposed indexes, the Gait Profile Score (GPS) proposed by Baker and colleagues [93] has quickly gained popularity, especially owing to its versatility which makes it employable in a wide range of neurologic and non-neurologic diseases. The GPS score, which is separately defined for each side of the body, is based upon nine key relevant kinematic variables, namely pelvic tilt, rotation and obliquity, hip flexion-extension, ad-abduction and rotation, knee flexion-extension, ankle dorsiflexion and foot progression. For each of them the root mean square (RMS) difference across the whole gait cycle is computed between the patient's data and the mean value extracted from a reference dataset obtained from healthy subjects. This value, which is referred to as Gait Variable Score (GVS), is calculated for each of the nine kinematic variables considered.

The GPS is thus expressed by the following relationship:

$$
\text { GPS }=\sqrt{\frac{1}{N} \sum_{i=1}^{N} \mathrm{GVS}_{1}^{2}}
$$

in which higher values of GPS represent larger deviations from a normal gait pattern.

In practice, while the GPS summarizes the overall deviation of the patient's gait pattern with respect to a healthy population, the GVS describes the specific alteration related to a certain movement of a joint. The GPS is usually graphically displayed in the form of a bar chart along with the nine GVSs: this representation is known as the Movement Analysis Profile (MAP). On the MAP, the height of each bar indicates the GVS score, and this provides quick visual information useful in assessing which variables may be responsible for high GPS values.

A limitation of this approach is represented by the fact that even though the values of GPS and GVS provide an effective idea of gait alterations, no information is available on the type of such alteration or the time of their occurrence in the gait cycle. For instance, an excess or a deficit in knee flexion may originate the same GVS value if the distance of the two curves from normality is the same.

In addition, the GPS, owing to its intrinsic nature of kinematic parameters, does not take into account the spatiotemporal variables of gait, which are equally important in defining the limitations associated with the pathology. For this reason, GPS and spatio-temporal values should be used in conjunction as complementary outcome measures for a global comprehension of the patient's gait limitations.

The use of GPS has been found to be effective in evaluating gait abnormalities in a wide spectrum of pathologies including Cerebral Palsy [93], Ehlers-Danlos syndrome [94], Multiple Sclerosis [95] and in different neurological and orthopedic diseases [96]. Moreover, strong, significant, positive correlations (i.e. Spearman correlations ranging from 0.84 to 0.97 ) were found between the GPS and GVS scores and clinicians' ratings of kinematic gait deviation [97].

Recently, the feasibility of applying the GPS/GVS approach has been tested with encouraging results among patients with PD. In particular, Speciali and colleagues propose the use of GPS to assess the effects of pharmacologic (levodopa) and neurosurgical (subthalamic deep brain stimulation, DBS) treatments of gait kinematics [98]. They detected significantly lower values of GPS in the case of DBS, mainly due to larger improvements exhibited in terms of hip flexion-extension with respect to levodopa. The GPS variations were also found to be substantially in agreement with previous similar studies in which kinematic changes were detected by analyzing the joint range of motion and the spatio-temporal parameters of gait (i.e. walking speed and stride/step length).

In another study by the same researchers [99], the GPS was employed to assess changes in gait kinematics in patients with PD when a concurrent cognitive task was added. They found that the GPS value increased under dual task conditions, thus indicating a significant alteration of gait pattern. In particular, all the GVS values increased, with the exception of hip rotation and foot progression.

Summarizing, these studies have shown that the GPS/GVS-based approach appears suitable not only in describing differences in gait patterns between individuals with PD and healthy subjects, but also in easily assessing the effectiveness of pharmacologic/rehabilitative treatments or the alterations in gait associated with the effect of specific cognitive loads.

\subsection{Kinetics and Muscular Activation}

In a modern laboratory for human movement analysis, kinematic data are routinely integrated with synchronized kinetic data (i.e. joint moments and powers) calculated on the basis of the force exchanged between the body and the ground which are collected using a force platform embedded in the walkway. The typical alterations of kinetic patterns in individuals with PD are represented in the form of a generalized reduction of the peaks of generated and absorbed mechanical power for hip, knee and ankle joints [82, 84, 87]. Interestingly, it has been shown that while a number of 
therapeutic treatments succeed in generally restoring knee power generation at values similar to non-affected subjects, reduced power levels persist at the ankle joint in the push-off phase and in the hip joint at pull off $[87,88]$.

Finally, GA can be optionally made still more accurate and complete by including in it the study of muscular activation patterns during gait, a task that can be performed using sEMG with either wired or wireless devices. The analyzed muscles are usually vastus lateralis, tibialis anterior, soleus and gastrocnemius, and the sEMG signal is explored to assess activation timing, symmetry between the two limbs and stride to stride variability $[28,84,100]$. It is to be noted that few existing studies analyzed sEMG data during gait. In particular, they focused on establishing differences between individuals with PD and healthy controls [28], on assessing effects of visual cues as tools to regulate stride length [84] and on investigating freezing episodes [100].

\subsection{Gait Analysis as a Tool to Verify the Effectiveness of the RAS Approach}

Since early studies in the mid-1990s, researchers have attempted to employ quantitative techniques to verify the effectiveness of the RAS approach in improving gait patterns of individuals with PD. Thaut et al. [29] analyzed sEMG and computerized foot-switch data to confirm the beneficent effect of RAS expressed through significant improvement in velocity, stride length and cadence, as well as in timing of tibialis anterior and vastus lateralis activation. To date, GA represents the gold standard for such evaluations and it has been used in most similar studies, even though mainly to assess spatio-temporal parameters and variability of gait [13, 101]. However, whether RAS training is able to improve joint kinematics or not remains still partly unexplored.

\section{NEW APPROACHES FOR INTERVENTION: THE USE OF ECOLOGICAL STIMULATION}

The sounds typically used in the studies on the RAS method are metronome tones, music or their combination, whose beats per minute are manipulated by experimenters $[13,27]$. Surprisingly, the effects of ecological auditory stimuli, such as the rhythmic sounds produced by human walking, are almost unexplored. In our opinion this is unfortunate and we will illustrate the empirical findings that encourage the investigation of ecological sounds of human walking for the modulation of gait in PD patients.

\subsection{Neurophysiological Bases}

One of the reasons that should drive researchers to investigate the effects of ecological rhythmic sounds on gait is the discovery of the so-called "mirror neurons" [102]. Mirror neurons are "a particular class of visuomotor neurons, originally discovered in area F5 of the monkey premotor cortex, that discharge both when the monkey does a particular action and when it observes another individual (monkey or human) doing a similar action" [102, p.169]. Brain imaging studies suggest the existence of a mirror neuron system also in humans, having its core in the inferior parietal lobule, in the precentral gyrus, and in the inferior frontal gyrus [103, 104]. Indeed, those regions activate both in response to ac- tion observation and during action planning and execution, and would represent the neurological basis for imitation learning.

Initially, the studies on mirror neurons focused on brain activation due to visual observation of actions. Subsequent studies investigated the effects of sounds associated with the execution of movements, and found results similar to those obtained in the visual modality: the administration of actionrelated sounds activates the mirror neuron system, both in monkeys [105] and humans [106-109]. Those studies demonstrated that the mirror neuron system is not limited to the visual modality: Such neurons code actions independently of whether they are performed, seen, or heard. The discovery of mirror neurons sensitive to ecological sounds of action arouses new challenges for clinical research: 1) Is it possible to benefit from the properties of the mirror neuron system to improve motor rehabilitation? 2) If so, would an Ecological Rhythmic Auditory Stimulation (E-RAS) be helpful for PD patients?

To date, we are far from responding to these questions, but we can advance two considerations. The first is that the use of E-RAS - that is, the administration of human footstep sounds, manipulating the beats per minute - would not differ from the "classic" RAS, in terms of gait temporal support. Indeed, E-RAS would provide external timing that facilitates the regulation of the patients' internal timing, exactly like RAS, thus functionally supporting the deficient activity of the basal ganglia. However, the administration of ecological footstep sounds would activate the mirror neuron system to produce a greater activation of those areas that control the motor production. The second consideration is that one of the main motor symptoms in PD patients, the freezing of gait, is correlated with frontal executive deficits and with tissue loss in the left inferior frontal gyrus, left precentral gyrus and left inferior parietal gyrus [110]. It is important to note that all these areas also belong to the mirror neuron system $[103,106]$. Therefore, if the functionality of these areas can be somehow triggered through E-RAS, then this kind of stimulation might determine positive effects on patients with freezing of gait.

\subsection{Theoretical Bases}

The discovery of the mirror neuron system has provided a solid neurophysiological basis to the Theory of Event Coding $[111,112]$, which theoretically postulates a common representational system for perception and action. According to this theory, the match between perceptual and motor experience is a core element for perceptual influences on motor processes (and vice versa). Indeed, the perception of actionrelated stimuli should evoke a representation of the action to be performed, and this representation should be further reinforced by previous motor experience of the same action. The synergic activation of representational codes coming from both the sensorial system and previous motor experience would determine an increased probability that individuals would follow the action-related stimuli features for performing their movements. On the contrary, action-unrelated stimuli would lack matching with previous motor experience and, as a consequence, would have a slighter influence on motor performance. 
According to the theory, in the case of ecological rhythmic auditory stimulation, the administration of human footstep sounds would evoke the representation of feature codes (such as cadence, step length, gender and weight of the walker, etc.) associated with the experience of walking. Nevertheless, the representation of walking - and the corresponding feature codes - is somehow pre-activated by previous individual motor experience. Therefore, the more the auditory stimulation matches previous motor experience, the more the features of auditory stimuli should affect the motor system and, consequently, the motor outcomes. Vice versa, the feature codes of artificial rhythmic auditory stimulation (i.e. metronome tones or music) would be represented in the common representational system, but they would not be reinforced by previous motor experience. As a consequence, artificial stimuli should be less effective than ecological stimuli. Undoubtedly, also artificial stimuli affect motor production, but it is reasonable to predict that their influences on motor processes should be slighter compared to those of more evocative ecological stimuli, such as footstep sounds.

\subsection{Empirical Evidence in Perceptual and Motor Studies}

The role of ecological sound of actions in brain activation and motor representation has been addressed by several studies on complex movements. For instance, Woods and colleagues found that listening to action-related sport sounds determines the activation of auditory and motor planning areas, depending on the experience in a specific sport [113]. In particular, they found that expert athletes showed greater activation than novices in the inferior frontal gyrus and the parietal operculum, when passively listening to familiar sport sounds. Other studies on motor imagery suggest that the inferior frontal gyrus is involved also during mental representation of action $[114,115]$. Therefore, in the study by Woods and colleagues, the exposition to familiar action-related sounds probably evoked a major representation of action in expert athletes compared to novices, owing to their motor experience.

The motor experience is also a crucial factor for the representation and the recognition of one's own movement through sound. Indeed, it has been demonstrated that people are able to discriminate between sounds associated with their own motor performances and sounds produced by other individuals in many different actions, such as clapping [116], sport performances $[117,118]$, and musical performances [119]. Again, such evidence has been interpreted as the result of the matching between the representation evoked by the sound, in terms of temporal factors or as a gestalt, and the previous motor experience.

Empirical evidence suggests that evocative action-related sounds can also affect motor outcomes. For instance, Cesari and colleagues found that natural-like skateboarding sounds cause muscle activation in expert skaters compared to inexpert individuals [120]. Moreover, other studies have shown that ecological sounds can guide complex sport actions and positively affect athletes' performances [121-123]. Finally, very recent findings have highlighted that ecological sounds of breathing can induce a standardization of breath duration in healthy participants, and demonstrated that the impact of ecological sounds is greater compared to that induced by artificial sounds [124].

\subsection{Empirical Evidence on Parkinson}

To the best of our knowledge, the empirical evidence regarding the effects of ecological sounds on the gait of PD patients is limited to the study by Young, Rodger, and Craig [125]. In three experiments, these authors compared the effects of ecological footstep sounds, metronome sounds and synthesized walking sounds on the gait of PD patients and healthy controls. The ecological sounds were obtained by recording the sounds produced by a healthy male walking with different step lengths and at different cadences on coarse gravel. Of the three experiments, the first is of particular interest for the aims of the present review since it addresses a comparison between ecological footstep sounds and RAS based on metronome sounds. In a real-time imitation task, the authors examined the ability of participants to use auditory information for guiding walking actions. The results revealed no differences between the two sounds concerning the percentage change in step length, compared to baseline trials without auditory cues. However, for PD patients, step length variability was significantly lower in the ecological sound condition compared to the metronome one; moreover, PD patients showed significantly reduced variability in step length within the ecological sound condition, compared to the control group. The latter results highlight the superiority of ecological sounds in promoting a reduction of variability in the step length of PD patients, which is a desirable outcome for interventions since it represents a regularization of PD patients' gait.

In the second and third experiments described by Young and colleagues [125], the ecological sound was compared with a synthesized sound derived from recordings of the kinetic interactions between the foot and the walking surface. In the second experiment, the procedure was the same as the first (the only difference was the use of synthesized sounds instead of the metronome) and results revealed that PD patients reduced their step length variability in both conditions, but they failed to adapt to the target step length within the synthesized sound condition, unless previously and explicitly instructed about the stimuli step length. Vice versa, in the ecological sound condition, participants adapted to the target step length even without any instruction. Finally, in the third experiment, the authors compared the effects of ecological and synthesized sounds in combination with imagery. The results confirmed that PD patients were not able to adapt their step length in the synthesized sound condition. Moreover, the results revealed that compared to the control group, in the synthesized sound condition the step length variability of PD patients was significantly reduced, but it increased in combination with imagery. Vice versa, the ecological sound promoted both the adaptation and the reduction of step length variability, also in combination with imagery.

Altogether, the results of Young and colleagues [125] suggest the superiority of ecological sounds in improving the gait parameters of PD patients against non-ecological sounds and thus represent an encouraging starting point for future research. 


\section{CONCLUSION}

Motor symptoms and in particular gait disturbances probably represent the main impairment in PD patients' daily lives and affect their overall quality of life. The pharmacological treatment of motor symptoms represents only a partial solution, which needs to be integrated with other approaches. Among them, physical therapy accompanied by rhythmic auditory stimulation has been proven effective in improving gait patterns. In the present work we have reviewed this approach, examining it from the cognitive, neurological and biomechanical perspectives, and have suggested new directions for future research, both for the assessment of gait disturbances and their treatment.

Our review has highlighted two main points that future studies should further address. The first regards the use of three-dimensional quantitative multifactorial analyses to accurately assess gait impairments in PD patients and the effects of RAS interventions. Indeed, most previous studies focused on the assessment of spatio-temporal parameters of gait, while only a few of them in literature analyzed kinematics, kinetics and muscular activation data simultaneously. Although this approach may result of difficult application in daily clinical evaluations owing to the large and complex amount of data produced, it may provide very accurate information for research, showing exactly "where", "when" and "how" interventions act on patients' gait. The second point regards the use of ecological rhythmic auditory stimulation to modulate the gait of PD patients. Indeed, the majority of previous studies used artificial sounds (i.e. metronome and/or music) as a pacemaker to provide rhythmic cues that facilitated patients' coordination during their walk. However, we have reviewed theoretical issues, neurophysiological bases and cognitive findings that highlight the impact of ecological sounds on motor representation and production, suggesting their potential superiority over artificial sounds. For the present, empirical evidence concerning the effects of ecological sound on PD patients' gait are very limited but, at the same time, they are very promising, thus we encourage investigating in this direction.

\section{CONFLICT OF INTEREST}

The authors confirm that this article content has no conflict of interest.

\section{ACKNOWLEDGEMENTS}

The author Mauro Murgia was supported by Autonomous Region of Sardinia, Master\&Back Programme 2013 (PRRMAB-A2013-19330).

\section{REFERENCES}

[1] Politis M, Niccolini F. Serotonin in Parkinson's disease. Behav Brain Res 2015; 277: 136-45.

[2] Beitz JM. Parkinson's disease: A review. Front Biosci Sch Ed 2014; 6: 65-74.

[3] Chaudhuri KR, Yates L, Martinez-Martin P. The non-motor symptom complex of Parkinson's disease: A comprehensive assessment is essential. Curr Neurol Neurosci Rep 2005; 5(4): 275-83.

[4] Politis M, Wu K, Molloy S, et al. Parkinson's disease symptoms: The patient's perspective. Mov Disord Off J Mov Disord Soc 2010; 25(11): 1646-51.
[5] Jellinger KA. Pathology of Parkinson's disease. Changes other than the nigrostriatal pathway. Mol Chem Neuropathol 1991; 14(3): 153-97.

[6] Brooks DJ, Piccini P. Imaging in Parkinson's disease: The role of monoamines in behavior. Biol Psychiatry 2006; 59(10): 908-18.

[7] Fox SH. Non-dopaminergic treatments for motor control in Parkinson's disease. Drugs 2013; 73(13): 1405-15.

[8] Kish SJ, Tong J, Hornykiewicz O, et al. Preferential loss of serotonin markers in caudate versus putamen in Parkinson's disease. Brain J Neurol 2008; 131(Pt 1): 120-31.

[9] Remy P, Doder M, Lees A, et al. Depression in Parkinson's disease: loss of dopamine and noradrenaline innervation in the limbic system. Brain 2005; 128(6): 1314-22.

[10] Uc EY, Doerschug KC, Magnotta V, et al. Phase I/II randomized trial of aerobic exercise in Parkinson disease in a community setting. Neurology 2014; 83(5): 413-25.

[11] Morris ME, Huxham F, McGinley J, et al. The biomechanics and motor control of gait in Parkinson disease. Clin Biomech Bristol Avon 2001; 16(6): 459-70.

[12] Blin O, Ferrandez AM, Serratrice G. Quantitative analysis of gait in Parkinson patients: Increased variability of stride length. J Neurol Sci 1990; 98(1): 91-7.

[13] Nombela C, Hughes LE, Owen AM, et al. Into the groove: Can rhythm influence Parkinson's disease? Neurosci Biobehav Rev 2013; 37(10, Pt 2): 2564-70.

[14] Jones CRG, Malone TJL, Dirnberger G, et al. Basal ganglia, dopamine and temporal processing: performance on three timing tasks on and off medication in Parkinson's disease. Brain Cogn 2008; 68(1): 30-41

[15] Ferrandez AM, Hugueville L, Lehéricy S, et al. Basal ganglia and supplementary motor area subtend duration perception: An fMRI study. NeuroImage 2003; 19(4): 1532-44.

[16] Harrington DL, Boyd LA, Mayer AR, et al. Neural representation of interval encoding and decision making. Brain Res Cogn Brain Res 2004; 21(2): 193-205.

[17] Jahanshahi M, Jones CRG, Dirnberger G, et al. The substantia nigra pars compacta and temporal processing. J Neurosci Off J Soc Neurosci 2006; 26(47): 12266-73.

[18] Nenadic I, Gaser C, Volz H-P, et al. Processing of temporal information and the basal ganglia: New evidence from fMRI. Exp Brain Res 2003; 148(2): 238-46.

[19] Rao SM, Mayer AR, Harrington DL. The evolution of brain activation during temporal processing. Nat Neurosci 2001; 4(3): 317-23.

[20] Harrington DL, Haaland KY, Hermanowicz N. Temporal processing in the basal ganglia. Neuropsychology 1998; 12(1): 3-12.

[21] Lange KW, Tucha O, Steup A, et al. Subjective time estimation in Parkinson's disease. J Neural Transm Suppl 1995; 46: 433-8.

[22] O'Boyle DJ, Freeman JS, Cody FW. The accuracy and precision of timing of self-paced, repetitive movements in subjects with Parkinson's disease. Brain J Neurol 1996; 119(Pt 1): 51-70.

[23] Pastor MA, Artieda J, Jahanshahi M, et al. Time estimation and reproduction is abnormal in Parkinson's disease. Brain J Neurol 1992; 115(Pt 1): 211-25.

[24] Malapani C, Rakitin B, Levy R, et al. Coupled temporal memories in Parkinson's disease: A dopamine-related dysfunction. J Cogn Neurosci 1998; 10(3): 316-31.

[25] Wearden JH, Smith-Spark JH, Cousins R, et al. Stimulus timing by people with Parkinson's disease. Brain Cogn 2008; 67(3): 264-79.

[26] Thaut MH, McIntosh GC, Prassas SG, et al. Effect of rhythmic auditory cueing on temporal stride parameters and EMG. Patterns in Hemiparetic Gait of Stroke Patients. Neurorehabil Neural Repair 1993; 7(1): 9-16.

[27] Thaut MH, Abiru M. Rhythmic auditory stimulation in rehabilitation of movement disorders: A review of current research. Music Percept 2010; 27(4): 263-9.

[28] Miller RA, Thaut MH, McIntosh GC, et al. Components of EMG symmetry and variability in parkinsonian and healthy elderly gait. Electroencephalogr Clin Neurophysiol 1996; 101(1): 1-7.

[29] Thaut MH, McIntosh GC, Rice RR, et al. Rhythmic auditory stimulation in gait training for Parkinson's disease patients. Mov Disord Off J Mov Disord Soc 1996; 11(2): 193-200. 
[30] Arias P, Cudeiro J. Effects of rhythmic sensory stimulation (auditory, visual) on gait in Parkinson's disease patients. Exp Brain Res 2008; 186(4): 589-601.

[31] Arias P, Cudeiro J. Effect of rhythmic auditory stimulation on gait in Parkinsonian Patients with and without freezing of gait. PLoS One 2010; 5(3): e9675.

[32] Baker K, Rochester L, Nieuwboer A. The immediate effect of attentional, auditory, and a combined cue strategy on gait during single and dual tasks in Parkinson's disease. Arch Phys Med Rehabil 2007; 88(12): 1593-600.

[33] Baker K, Rochester L, Nieuwboer A. The effect of cues on gait variability--reducing the attentional cost of walking in people with Parkinson's disease. Parkinsonism Relat Disord 2008; 14(4): 31420.

[34] Enzensberger W, Fischer PA. Metronome in Parkinson's disease. Lancet 1996; 347(9011): 1337.

[35] Fernandez del OM, Cudeiro J. A simple procedure using auditory stimuli to improve movement in Parkinson's disease: A pilot study. Neurol Clin Neurophysiol NCN 2003; 2003(2): 1-7.

[36] Freedland RL, Festa C, Sealy M, et al. The effects of pulsed auditory stimulation on various gait measurements in persons with Parkinson's Disease. NeuroRehabilitation 2002; 17(1): 81-7.

[37] Hove MJ, Suzuki K, Uchitomi H, et al. Interactive rhythmic auditory stimulation reinstates natural 1/f timing in gait of Parkinson's Patients. PLoS One 2012; 7(3): e32600.

[38] Howe TE, Lövgreen B, Cody FWJ, et al. Auditory cues can modify the gait of persons with early-stage Parkinson's disease: A method for enhancing parkinsonian walking performance? Clin Rehabil 2003; 17(4): 363-7.

[39] Jiang Y, Norman KE. Effects of visual and auditory cues on gait initiation in people with Parkinson's disease. Clin Rehabil 2006; 20(1): 36-45.

[40] McIntosh GC, Brown SH, Rice RR, et al. Rhythmic auditory-motor facilitation of gait patterns in patients with Parkinson's disease. J Neurol Neurosurg Psychiatry 1997; 62(1): 22-6.

[41] Nanhoe-Mahabier W, Delval A, Snijders AH, et al. The possible price of auditory cueing: Influence on obstacle avoidance in Parkinson's disease. Mov Disord Off J Mov Disord Soc 2012; 27(4): 574-8.

[42] Nieuwboer A, Baker K, Willems A-M, et al. The short-term effects of different cueing modalities on turn speed in people with Parkinson's disease. Neurorehabil Neural Repair 2009; 23(8): 831-6.

[43] Rochester L, Hetherington V, Jones D, et al. The effect of external rhythmic cues (auditory and visual) on walking during a functional task in homes of people with Parkinson's disease. Arch Phys Med Rehabil 2005; 86(5): 999-1006.

[44] Rochester L, Burn DJ, Woods G, et al. Does auditory rhythmical cueing improve gait in people with Parkinson's disease and cognitive impairment? A feasibility study. Mov Disord Off J Mov Disord Soc 2009; 24(6): 839-45.

[45] Suteerawattananon M, Morris GS, Etnyre BR, et al. Effects of visual and auditory cues on gait in individuals with Parkinson's disease. J Neurol Sci 2004; 219(1-2): 63-9.

[46] Willems AM, Nieuwboer A, Chavret F, et al. The use of rhythmic auditory cues to influence gait in patients with Parkinson's disease, the differential effect for freezers and non-freezers, an explorative study. Disabil Rehabil 2006; 28(11): 721-8.

[47] Willems A-M, Nieuwboer A, Chavret F, et al. Turning in Parkinson's disease patients and controls: The effect of auditory cues. Mov Disord Off J Mov Disord Soc 2007; 22(13): 1871-8.

[48] Zijlstra W, Rutgers A, Van Weerden TW. Voluntary and involuntary adaptation of gait in Parkinson's disease. Gait Posture 1998; 7(1): 53-63.

[49] Cubo E, Leurgans S, Goetz CG. Short-term and practice effects of metronome pacing in Parkinson's disease patients with gait freezing while in the "on" state: Randomized single blind evaluation. Parkinsonism Relat Disord 2004; 10(8): 507-10.

[50] Fernandez del Olmo M, Cudeiro J. Temporal variability of gait in Parkinson disease: Effects of a rehabilitation programme based on rhythmic sound cues. Parkinsonism Relat Disord 2005; 11(1): 2533 .
[51] Fernandez del OM, Arias P, Furio MC, et al. Evaluation of the effect of training using auditory stimulation on rhythmic movement in Parkinsonian patients--a combined motor and [18F]-FDG PET study. Parkinsonism Relat Disord 2006; 12(3): 155-64.

[52] Ford MP, Malone LA, Nyikos I, et al. Gait training with progressive external auditory cueing in persons with Parkinson's disease. Arch Phys Med Rehabil 2010; 91(8): 1255-61.

[53] Frazzitta G, Maestri R, Uccellini D, et al. Rehabilitation treatment of gait in patients with Parkinson's disease with freezing: A comparison between two physical therapy protocols using visual and auditory cues with or without treadmill training. Mov Disord Off J Mov Disord Soc 2009; 24(8): 1139-43.

[54] Kadivar Z, Corcos DM, Foto J, et al. Effect of step training and rhythmic auditory stimulation on functional performance in Parkinson patients. Neurorehabil Neural Repair 2011; 25(7): 626-35.

[55] Lim I, van Wegen E, Jones D, et al. Does cueing training improve physical activity in patients with Parkinson's disease? Neurorehabil Neural Repair 2010; 24(5): 469-77.

[56] Marchese R, Diverio M, Zucchi F, et al. The role of sensory cues in the rehabilitation of parkinsonian patients: A comparison of two physical therapy protocols. Mov Disord Off J Mov Disord Soc 2000; 15(5): 879-83.

[57] Nieuwboer A, Kwakkel G, Rochester L, et al. Cueing training in the home improves gait-related mobility in Parkinson's disease: The RESCUE trial. J Neurol Neurosurg Psychiatry 2007; 78(2): 134-40.

[58] Rochester L, Nieuwboer A, Baker K, et al. The attentional cost of external rhythmical cues and their impact on gait in Parkinson's disease: effect of cue modality and task complexity. J Neural Transm Vienna Austria 1996; 114(10): 1243-8.

[59] Rochester L, Baker K, Hetherington V, et al. Evidence for motor learning in Parkinson's disease: Acquisition, automaticity and retention of cued gait performance after training with external rhythmical cues. Brain Res 2010; 1319: 103-11.

[60] Hausdorff JM, Lowenthal J, Herman T, et al. Rhythmic auditory stimulation modulates gait variability in Parkinson's disease. Eur J Neurosci 2007; 26(8): 2369-75.

[61] Thaut MH, Miller RA, Schauer LM. Multiple synchronization strategies in rhythmic sensorimotor tasks: phase vs period correction. Biol Cybern 1998; 79(3): 241-50.

[62] Thaut MH, Kenyon GP, Schauer ML, et al. The connection between rhythmicity and brain function. IEEE Eng Med Biol Mag 1999; 18(2): 101-8.

[63] Lim I, van Wegen E, de Goede C, et al. Effects of external rhythmical cueing on gait in patients with Parkinson's disease: A systematic review. Clin Rehabil 2005; 19(7): 695-713.

[64] Giladi N, Fahn S. Freezing phenomenon, the fifth cardinal sign of Parkinsonism. In: Fisher A, Hanin I, Yoshida M, Eds. Progress in Alzheimer's and Parkinson's Diseases. US: Springer 1998; pp. 329-35.

[65] Chen JL, Penhune VB, Zatorre RJ. Listening to musical rhythms recruits motor regions of the brain. Cereb Cortex 2008; 18(12): 2844-54.

[66] Grahn JA, Brett M. Rhythm and beat perception in motor areas of the brain. J Cogn Neurosci 2007; 19(5): 893-906.

[67] Stupacher J, Hove MJ, Novembre G, et al. Musical groove modulates motor cortex excitability: A TMS investigation. Brain Cogn 2013; 82(2): 127-36

[68] Grondin S, McAuley D. Duration discrimination in crossmodal sequences. Perception 2009; 38(10): 1542-59.

[69] Grondin S. Timing and time perception: A review of recent behavioral and neuroscience findings and theoretical directions. Atten Percept Psychophys 2010; 72(3): 561-82.

[70] Repp BH, Penel A. Auditory dominance in temporal processing: new evidence from synchronization with simultaneous visual and auditory sequences. J Exp Psychol Hum Percept Perform 2002; 28(5): 1085-99.

[71] Repp BH, Penel A. Rhythmic movement is attracted more strongly to auditory than to visual rhythms. Psychol Res 2004; 68(4): 25270 . 
[72] Mushiake H, Inase M, Tanji J. Selective coding of motor sequence in the supplementary motor area of the monkey cerebral cortex. Exp Brain Res 1990; 82(1): 208-10.

[73] Thaut MH, McIntosh KW, McIntosh GC, et al. Auditory rhythmicity enhances movement and speech motor control in patients with Parkinson's disease. Funct Neurol 2001; 16(2): 163-72.

[74] Hallett M. The intrinsic and extrinsic aspects of freezing of gait. Mov Disord Off J Mov Disord Soc 2008; 23(Suppl 2): S439-43.

[75] Jahanshahi M, Jenkins IH, Brown RG, et al. Self-initiated versus externally triggered movements. I. An investigation using measurement of regional cerebral blood flow with PET and movementrelated potentials in normal and Parkinson's disease subjects. Brain J Neurol 1995; 118(Pt 4): 913-33.

[76] Wu T, Chan P, Hallett M. Effective connectivity of neural networks in automatic movements in Parkinson's disease. NeuroImage 2010; 49(3): 2581-7.

[77] Knutsson E, Mårtensson A. Quantitative effects of L-dopa on different types of movements and muscle tone in Parkinsonian patients. Scand J Rehabil Med 1971; 3(3): 121-30.

[78] Knutsson E. An analysis of Parkinsonian gait. Brain J Neurol 1972; 95(3): 475-86.

[79] Murray MP, Sepic SB, Gardner GM, et al. Walking patterns of men with parkinsonism. Am J Phys Med 1978; 57(6): 278-94.

[80] Ferrari A, Benedetti MG, Pavan E, et al. Quantitative comparison of five current protocols in gait analysis. Gait Posture 2008; 28(2): 207-16.

[81] Defebvre L, Blatt JL, Blond S, et al. Effect of thalamic stimulation on gait in Parkinson disease. Arch Neurol 1996; 53(9): 898-903.

[82] Morris ME, McGinley J, Huxham F, et al. Constraints on the kinetic, kinematic and spatiotemporal parameters of gait in Parkinson's disease. Hum Mov Sci 1999; 18(2-3): 461-83.

[83] Grasso R, Peppe A, Stratta F, et al. Basal ganglia and gait control: Apomorphine administration and internal pallidum stimulation in Parkinson's disease. Exp Brain Res 1999; 126(2): 139-48.

[84] Lewis GN, Byblow WD, Walt SE. Stride length regulation in Parkinson's disease: The use of extrinsic, visual cues. Brain J Neurol 2000; 123(Pt 10): 2077-90.

[85] Shan DE, Lee SJ, Chao LY, et al. Gait analysis in advanced Parkinson's disease-effect of levodopa and tolcapone. J Can Sci Neurol 2001; 28(1): 70-5.

[86] Morris M, Iansek R, McGinley J, et al. Three-dimensional gait biomechanics in Parkinson's disease: evidence for a centrally mediated amplitude regulation disorder. Mov Disord Off J Mov Disord Soc 2005; 20(1): 40-50.

[87] Ferrarin M, Rizzone M, Bergamasco B, et al. Effects of bilateral subthalamic stimulation on gait kinematics and kinetics in Parkinson's disease. Exp Brain Res 2005; 160(4): 517-27.

[88] Sofuwa O, Nieuwboer A, Desloovere K, et al. Quantitative gait analysis in Parkinson's disease: comparison with a healthy control group. Arch Phys Med Rehabil 2005; 86(5): 1007-13.

[89] Peppe A, Chiavalon C, Pasqualetti P, et al. Does gait analysis quantify motor rehabilitation efficacy in Parkinson's disease patients? Gait Posture 2007; 26(3): 452-62.

[90] Roiz R de M, Cacho EWA, Pazinatto MM, et al. Gait analysis comparing Parkinson's disease with healthy elderly subjects. Arq Neuropsiquiatr 2010; 68(1): 81-6.

[91] Ayán C, Cancela JM, Gutiérrez-Santiago A, et al. Effects of two different exercise programs on gait parameters in individuals with Parkinson's disease: A pilot study. Gait Posture 2014; 39(1): 64851.

[92] Cimolin V, Galli M. Summary measures for clinical gait analysis: A literature review. Gait Posture 2014; 39(4): 1005-10.

[93] Baker R, McGinley JL, Schwartz MH, et al. The gait profile score and movement analysis profile. Gait Posture 2009; 30(3): 265-9.

[94] Celletti C, Galli M, Cimolin V, et al. Use of the gait profile score for the evaluation of patients with joint hypermobility syndrome/Ehlers-Danlos syndrome hypermobility type. Res Dev Disabil 2013; 34(11): 4280-5.

[95] Pau M, Coghe G, Atzeni C, et al. Novel characterization of gait impairments in people with multiple sclerosis by means of the gait profile score. J Neurol Sci 2014; 345(1-2): 159-63.
[96] Schweizer K, Romkes J, Coslovsky M, et al. The influence of muscle strength on the gait profile score (GPS) across different patients. Gait Posture 2014; 39(1): 80-5.

[97] Beynon S, McGinley JL, Dobson F, et al. Correlations of the gait profile score and the movement analysis profile relative to clinical judgments. Gait Posture 2010; 32(1): 129-32.

[98] Speciali DS, Corrêa JCF, Luna NM, et al. Validation of GDI, GPS and GVS for use in Parkinson's disease through evaluation of effects of subthalamic deep brain stimulation and levodopa. Gait Posture 2014; 39(4): 1142-5.

[99] Speciali DS, Oliveira EM, Cardoso JR, et al. Gait profile score and movement analysis profile in patients with Parkinson's disease during concurrent cognitive load. Braz J Phys Ther 2014; 18(4): 31522.

[100] Nieuwboer A, Dom R, De Weerdt W, et al. Electromyographic profiles of gait prior to onset of freezing episodes in patients with Parkinson's disease. Brain J Neurol 2004; 127(Pt 7): 1650-60.

[101] Dalla Bella S, Benoit C-E, Farrugia N, et al. Effects of musically cued gait training in Parkinson's disease: Beyond a motor benefit Ann N Y Acad Sci 2015; 1337: 77-85.

[102] Rizzolatti G, Fadiga L, Gallese V, et al. Premotor cortex and the recognition of motor actions. Brain Res Cogn Brain Res 1996; 3(2): 131-41.

[103] Rizzolatti G, Craighero L. The mirror-neuron system. Annu Rev Neurosci 2004; 27: 169-92.

[104] Rizzolatti G, Fadiga L, Matelli M, et al. Localization of grasp representations in humans by PET: 1. Observation versus execution. Exp Brain Res 1996; 111(2): 246-52.

[105] Kohler E, Keysers C, Umiltà MA, et al. Hearing sounds, understanding actions: Action representation in mirror neurons. Science 2002; 297(5582): 846-8.

[106] Galati G, Committeri G, Spitoni G, et al. A selective representation of the meaning of actions in the auditory mirror system. NeuroImage 2008; 40(3): 1274-86.

[107] Gazzola V, Aziz-Zadeh L, Keysers C. Empathy and the somatotopic auditory mirror system in humans. Curr Biol CB 2006; 16(18): 1824-9.

[108] Lewis JW, Brefczynski JA, Phinney RE, et al. Distinct cortical pathways for processing tool versus animal sounds. J Neurosci 2005; 25(21): 5148-58.

[109] Tettamanti M, Buccino G, Saccuman MC, et al. Listening to action-related sentences activates fronto-parietal motor circuits. J Cogn Neurosci 2005; 17(2): 273-81.

[110] Kostic VS, Agosta F, Pievani M, et al. Pattern of brain tissue loss associated with freezing of gait in Parkinson disease. Neurology 2012; 78(6): 409-16.

[111] Hommel B, Müsseler J, Aschersleben G, et al. The Theory of Event Coding (TEC): A framework for perception and action planning. Behav Brain Sci 2001; 24(5): 849-78.

[112] Zmigrod S, Hommel B. Auditory event files: Integrating auditory perception and action planning. Atten Percept Psychophys 2009; 71(2): 352-62.

[113] Woods EA, Hernandez AE, Wagner VE, et al. Expert athletes activate somatosensory and motor planning regions of the brain when passively listening to familiar sports sounds. Brain Cogn 2014; 87: 122-33.

[114] Mizuguchi N, Nakata H, Kanosue K. Effector-independent brain activity during motor imagery of the upper and lower limbs: An fMRI study. Neurosci Lett 2014; 581: 69-74.

[115] Taube W, Mouthon M, Leukel C, et al. Brain activity during observation and motor imagery of different balance tasks: An fMRI study. Cortex 2015; 64: 102-14.

[116] Flach R, Knoblich G, Prinz W. Recognizing one's own clapping: the role of temporal cues. Psychol Res 2004; 69(1-2): 147-56.

[117] Kennel C, Pizzera A, Hohmann T, et al. The perception of natural and modulated movement sounds. Perception 2014; 43(8): 796804.

[118] Murgia M, Hohmann T, Galmonte A, et al. Recognising one's own motor actions through sound: The role of temporal factors. Perception 2012; 41(8): 976-87.

[119] Repp BH, Knoblich G. Perceiving action identity: How pianists recognize their own performances. Psychol Sci 2004; 15(9): 604-9. 
[120] Cesari P, Camponogara I, Papetti S, et al. Might as well jump: Sound affects muscle activation in skateboarding. PLoS One 2014; 9(3): e90156.

[121] Agostini T, Righi G, Galmonte A, et al. The relevance of auditory information in optimizing hammer throwers performance. In: Pascolo PB, Ed. Biomechanics and Sports. Vienna: Springer 2004; pp. 67-74.

[122] Righi G, Ferletic E, Furlan D, et al. Are visual models the best models to learn a specific task in sport training? Perception 2007; 36: 179.
[123] Murgia M, Bresolin G, Righi G, et al. The effect of visual and auditory models on golf swing. J Sport Exerc Psychol 2011; 33: S91.

[124] Murgia M, Santoro I, Tamburini G, et al. Ecological sounds affect breath duration more than artificial sounds. Psychol Res 2015; [epub ahead of print]. DOI 10.1007/s00426-015-0647-z.

[125] Young WR, Rodger MWM, Craig CM. Auditory observation of stepping actions can cue both spatial and temporal components of gait in Parkinson's disease patients. Neuropsychologia 2014; 57: 140-53.

(C) Murgia et al.; Licensee Bentham Open.

This is an open access articles licensed under the terms of the Creative Commons Attribution-Non-Commercial 4.0 International Public License (CC BY-NC 4.0) (https://creativecommons.org/licenses/by-nc/4.0/legalcode), which permits unrestricted, non-commercial use, distribution and reproduction in any medium, provided that the work is properly cited. 